\title{
Terminal
}

Technologie de l'information, culture \& société

120 | 2017 :

Les enjeux de la transformation numérique dans l'entreprise du futur

Les enjeux de la transformation numérique dans l'entreprise du futur

\section{Les usages des outils de réseau social par des salariés : Des registres privés et professionnels individualisés}

Employees' Uses of Social Network Sites: Individualised Private and Professional Dimensions

KARINE ROUDAUT ET NICOLAS JULLIEN

\section{Résumés}

\section{Français English}

Cet article sur les usages des outils de réseau social par les salariés s'inscrit dans une tradition de recherche sur les effets de la technologie sur le travail, sur l'évolution des frontières vie privée et vie professionnelle. Il questionne la façon dont ils gèrent leurs identités (privée, publique, professionnelle, personnelle). Menée dans une grande entreprise française du secteur des TIC, l'enquête - quantitative et qualitative - souligne premièrement, l'existence d'une conscience forte du caractère professionnel et privé de ces outils; deuxièmement, un usage spécifique des plateformes: une tentative de cloisonnement. Le mélange des usages des outils est à l'image de l'entrelacement des sociabilités dans la vie hors ligne.

This article addresses the question of employees' perceived usefulness of social networking sites in their professional activity. The quantitative and qualitative survey conducted in a large French company from the ICT sector, shows a low level professional use of online social sites. We identify two types of users, associated with types of use and types of networks. If the users creates the usage, it is individualized and disaffiliated from the company.

\section{Entrées d'index}

Mots-clés : réseaux sociaux numériques, usages des TIC, activité professionnelle, vie privée/publique, sociologie

Keywords : social network sites, professional activities, privacy, public/private life, sociology

\section{Texte intégral}




\section{Introduction}

Les réseaux sociaux numériques, et avant eux les forums, l'intranet, le courriel, redéfinissent des temporalités et la production du travail en déplaçant, voire mêlant les frontières traditionnelles de nos mondes sociaux, familial et privé, personnel et professionnel $^{1}$. Cet effet de porosité ou de perméabilité des mondes et des identités apparaît prégnant depuis l'arrivée du Web 2.0 (Georges, 2009), défini comme le Web relationnel (Kessous \& Rey, 2009).

2 Mais l'exposition sur ces sites qu'implique l'interaction sociale est potentiellement à risque pour le salarié qui peut y dévoiler des aspects de sa personnalité ou des opinions, qui ne correspondraient pas aux attentes de l'employeur (Gross et Acquisti, 2005).

3 À partir de l'observation des traces d'actions des individus sur les sites en ligne, $\mathrm{F}$. Georges (2009), après D. Cardon (2008), souligne l'effet d'une pression à l'exposition sur les comportements d'affichage des individus sur ces espaces numériques. Mais au-delà de la description d'activités extrêmes (Granjon et Denouël, 2010, Granjon, 2011) ou d'enquête auprès de publics étudiants (Ellison et al., 2007), peu se sont intéressés à la réflexivité de ces pratiques, aux stratégies quand elles existent, pour s'inventer (Kaufmann, 2004) ou conserver « un soi consistant et autonome » (Georges, 2009), y compris professionnel.

$4 \quad$ Partant du point de vue des utilisateurs, de leurs usages professionnels et privés de ces outils de réseau social (pratiques et utilité perçue), ce texte interroge la frontière entre les espaces privé et professionnel. Fait-elle sens pour les acteurs ? La préservent-ils ? Ou au contraire, l'intrication des espaces est-elle devenue une norme d'usage ? Après quelques résultats de la littérature sur ces réseaux dans le monde professionnel nous permettant de définir les axes d'analyse, et une présentation des corpus et méthodes de l'enquête (section 2), nous analysons les résultats en termes de segmentation des usages privés et professionnels des réseaux sociaux numériques (sections 3 et 4), avant de discuter l'idée d'individualisation et d'imbrication ou «entrelacement des usages » (Beaudoin et Velkovska, 1999) de l'individu, en tant que personne privée et professionnelle, ce que nous avons appelé « les frontières floues de l'identité » (section 5), et de conclure sur ce que cela indique dans l'évolution du soi au travail.

\section{Les outils de réseau social, le travail, la vie privée et l'enquête}

\section{Revue de la littérature}

La revue de la littérature de Glynn et al. (2012) caractérise les utilisateurs d'outils de réseau social : jeunes, extrovertis et à l'aise avec les nouvelles technologies. Pour l'usage professionnel, Morris et al. (2010), interrogeant des salariés de Microsoft sur l'intérêt pour ces outils, montrent que l'accès rapide à de l'expertise et la possibilité de développer des contacts justifient leur recours. Pour Thom-Santelli et al. (2011), analysant les usages d'un réseau social interne à une entreprise, les deux groupes d'employés le valorisant sont les nouveaux arrivants et les personnes éloignées des centres de décision pour acquérir ou conserver la culture de l'entreprise. Sur la question de l'intégration des personnels, Steinfield et al. (2009) relient l'usage de ces outils au sentiment d'appartenance à l'entreprise et à la possibilité d'accès direct à des personnes compétentes. Enfin, les créateurs d'un réseau social à HP défendent l'idée que cet outil interne a permis aux utilisateurs de se sentir plus « reliés » à l'entreprise et aux autres salariés et les a rendus plus attentifs à ce qui se passe dans les autres services (Brzozowski, 2009). L'outil y est vu comme un support de «la coopération dans des situations de travail » (Cardon, 1997), de 
la construction de communautés de pratique, internes à l'entreprise, et surtout comme un moyen de renforcer les solidarités internes ou liens professionnels, jusqu'à favoriser le développement d'un sentiment d'appartenance à une culture d'entreprise. Ce qui est cependant à nuancer.

$6 \quad$ Les employés sélectionnent des contacts professionnels au profil proche, percevant surtout les dimensions personnelles (Wu et al., 2010). Skeels et Grudin (2009) constatent un fort usage des sites de réseau social dans l'entreprise étudiée (Microsoft), mais pointent l'existence de tensions dans le mélange des connexions (professionnelles et personnelles) et dans le dépassement des limites de l'organisation.

7 Signe d'une injonction paradoxale des entreprises qui, d'un côté, voient dans le Web relationnel un moyen de favoriser des situations sociales et organisationnelles nouvelles au travail (Beaudouin et al., 2001 ; Muhlmann, 2001; Mallard, 2014) - par la mise en relation de ses employés -, en développant des communautés de pratiques, mais, d'un autre côté, tiennent à préserver leurs frontières organisationnelles. On sait en effet que les personnes s'impliquent dans ces communautés parce qu'elles ont des objectifs personnels, des besoins auxquels celles-ci répondent (Jullien et al., 2011). Le développement de liens sociaux arrive en sus. L'émergence d'un sentiment d'appartenance n'est le fait que des plus impliqués²

Suivant ces résultats, notre enquête avait pour objectif de cerner comment les individus gèrent leur activité sur les outils de réseau social numérique, afin d'intégrer la dimension professionnelle et l'effet prescriptif des entreprises. Suivant Karoui et Dudezert (2012), nous avons interrogé des salariés sur leur rapport aux outils de réseau social, en prenant en compte la maîtrise cognitive et technique des outils (ce qu'ils permettent et ce que les usagers en comprennent) et ce qu'ils en font dans leur vie professionnelle ou non, au travail ou en dehors. Pour répondre à la question de l'appropriation (Benghozi 2004; Cardon, 2004) et de la gestion de "l'entrelacement des usages", nous avons cherché à comprendre la signification des non-usages volontaires (Satchell et Dourish, 2009 ; Jauréguiberry, 2014) : refus d'afficher ou de rendre publiques des relations privées et professionnelles (Bateman et al., 2011) ou le manque d'intérêt perçu dans l'exercice de son métier et qui peut aussi renvoyer à une surcharge au travail.

\section{Corpus et méthodes d'enquête}

Le matériau produit provient de l'analyse de données quantitatives et discursives. Quelque 9000 salariés ont répondu à un questionnaire en ligne adressé de la mi-février au début de mars 2011 à l'ensemble des 100000 salariés de l'entreprise (taux de réponse de $9 \% ; 62 \%$ d'hommes, $38 \%$ de femmes) ${ }^{3}$, avec une forte surreprésentation des salariés jeunes 4 dans l'échantillon. Tous les niveaux hiérarchiques et les métiers de l'entreprise sont représentés5. 48,6 \% répondent être inscrits sur un réseau social. Cette inscription est plutôt récente ${ }^{6}$.

10 À la suite de ce questionnaire, nous avons réalisé 20 entretiens semi-directifs auprès de salariés de l'entreprise, recrutés parmi ceux qui avaient indiqué dans le questionnaire qu'ils étaient prêts à répondre à un entretien, ce qui nécessitait de laisser une adresse électronique pour être recontacté. À la suite du questionnaire, plus de 800 personnes avaient laissé leurs coordonnées en vue d'une interview.

11 Nous avons sélectionné uniquement des usagers de réseaux sociaux numériques. Certains étaient inscrits depuis peu de temps (quatre mois), d'autres depuis le début de ces outils (environ dix ans). Les répondants avaient entre 20 et 58 ans, faisaient partie de l'entreprise depuis en moyenne une dizaine d'années. Ils sont diplômés de formation supérieure (bac + 2-3 à post-doctorat), ce qui a une incidence sur les modes d'usage, sur la conscience des risques associés à la non-maîtrise de la technique et de l'usage, sur la capacité à gérer les différents contextes sociaux de leurs usages et les discours appropriés à ces contextes.

Le guide préalablement construit reprend les dimensions suivantes : le déroulement ou la trajectoire d'usage des outils de réseau social numériques, les activités particulières sur les différents outils, les contacts, les usages, ainsi que des éléments de parcours professionnel et 
de leur poste. Les entretiens ont presque tous été réalisés par téléphone; un entretien a été mené en face-à-face. Ils ont une durée de trente-cinq minutes à deux heures vingt.

\section{Ce qui fait l'inscription}

L’inscription sur un outil de réseau social concerne moins de la moitié des salariés de l'entreprise en 2011. Cette situation a sans doute un peu évolué.

Nous avons réalisé une régression logistique sur les facteurs expliquant l'inscription sur les réseaux (voir annexe 2). Il en ressort, de façon classique, que les jeunes ont plus de probabilité d'être inscrits ; un faible niveau d'étude diminue cette probabilité.

15 Plus inattendu, l'impact de la situation professionnelle est faible. À part l'ancienneté, les raisons évoquées par Thom-Santelli et al. (2011) (être en télétravail, à temps incomplet) ne jouent pas; disposer d'un ordinateur professionnel individuel non plus et l'accès à Internet dans l'entreprise diminue même la probabilité d'être connecté !

16 La recherche de solidarité professionnelle et l'outil de réseau social pour consolider son appartenance à l'entreprise ne semblent pas être des éléments forts de l'inscription.

17 Les principales causes professionnelles d'inscription sont à chercher dans les habitudes d'échange qui existaient avant ces outils : les personnes dont le domaine professionnel est $a$ priori proche des usages permis par les outils de réseau social (communication, marketing, innovation) ont plus de chance d'y être ; a contrario celles qui ne communiquent pas, n'interviennent pas sur des forums, ne consultent pas des sites Web externes, ont une probabilité plus faible.

18 Toujours pour souligner l'importance des caractéristiques personnelles, plutôt que d'entreprise pour expliquer l'inscription, l'impression de maitrise de l'identité professionnelle en ligne joue peu dans l'explication de l'inscription, même si elle souligne un paradoxe : ceux qui se disent très à l'aise avec ces outils ont moins de chance d'y être que ceux qui le sont moyennement. Ceci pointe la difficulté à estimer les risques de la présence sur ces réseaux, notamment en termes de vie privée, point développé dans l'analyse qualitative.

19 Finalement, les caractéristiques du métier des répondants, leur profil et compétences professionnelles sont plus importants que leur position dans l'entreprise pour expliquer l'intérêt pour de tels outils. Ce sont plus les compétences individuelles sollicitées par l'activité professionnelle (comme le fait de discuter, de se créer des réseaux) qui expliquent l'usage des outils de réseau social numérique.

\section{Une segmentation des mondes par l'outil}

Partant des inscrits, c'est-à-dire de 4187 réponses à l'enquête quantitative et des 20 entretiens, plusieurs points d'analyse ressortent.

L'outil de réseau social majoritairement utilisé est Facebook ; les autres le sont par une faible minorité des inscrits (illustration 1).

Illustration 1 : Les usages suivant les outils 


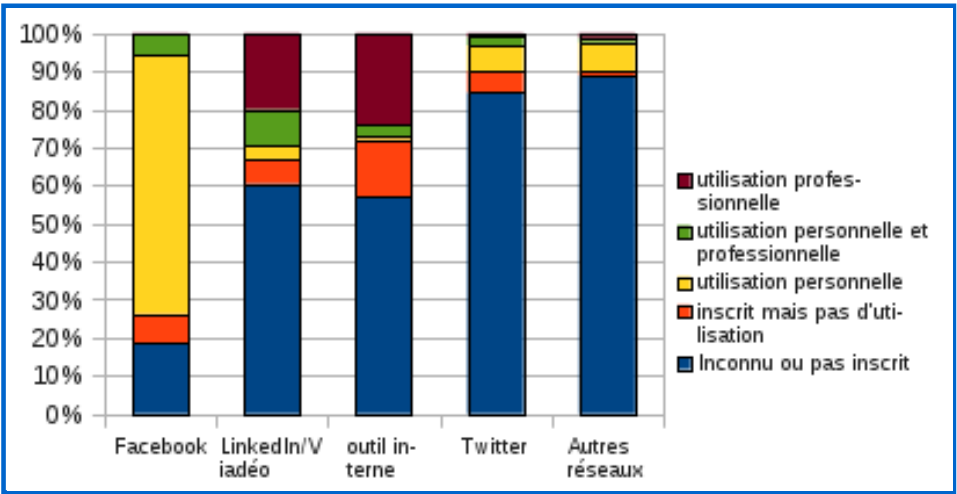

Chaque outil de réseau social renvoie à un réseau spécifique de contacts, auquel des usages spécifiques, professionnels ou personnels sont associés. Chaque outil identifie donc des utilisateurs ayant un profil précis. Ces résultats ressortent clairement des tris croisés entre les outils et les types d'usage (illustrations 2 et 3).

Illustration 2 : Les répondants, pour chaque outil, suivant les personnes avec qui ils échangent

Pour échanger des messages avec des collègues :

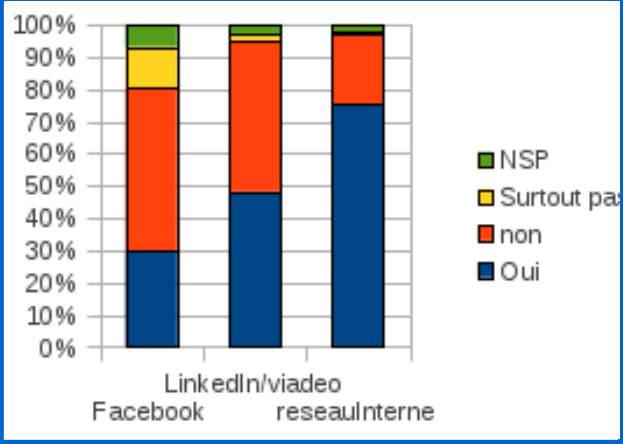

Pour échanger des messages avec des amis hors travail :

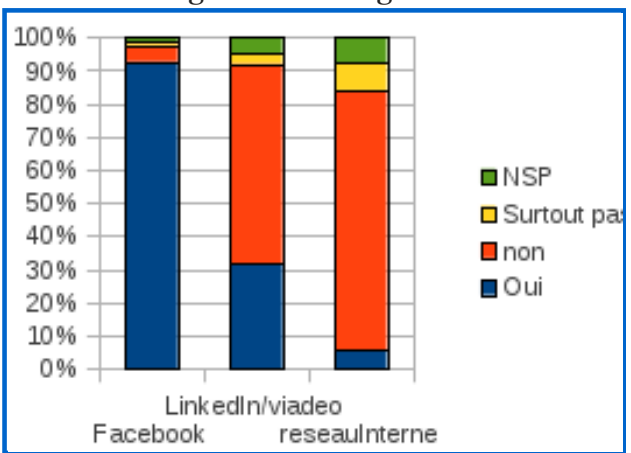

Illustration 3 : Les répondants, pour chaque outil, suivant les données publiées

Des données personnelles :

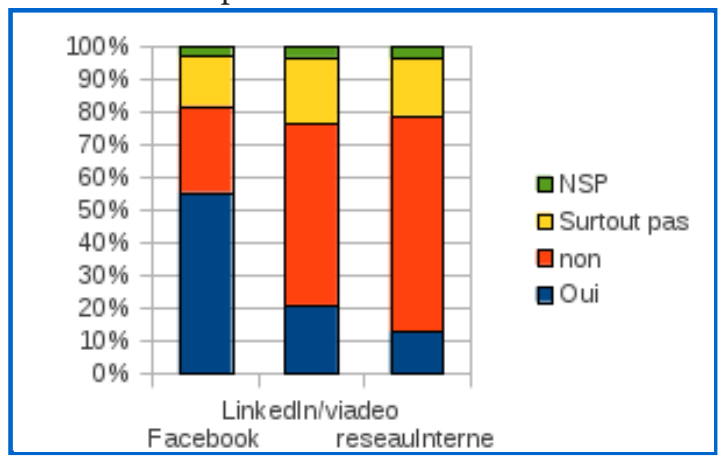

Des données professionnelles : 


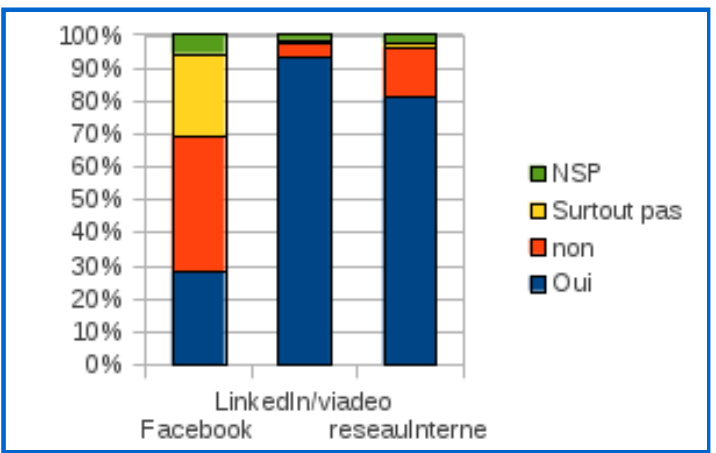

Ce résultat est confirmé par l'ACM et la classification ascendante hiérarchique que nous avons réalisées sur l'usage des différents outils de réseau social. Les variables actives sont celles d'utilisation des sites de réseau social (inscription ou non sur réseau social interne, Facebook, Viadeo/LinkedIn, utilisation professionnelle ou personnelle de ces mêmes réseaux), l'ancienneté de l'inscription sur ces sites, ainsi que des questions sur ce que les répondants font sur ces réseaux sociaux (présenté dans les illustrations) et, notamment, leurs usages professionnels de ces réseaux.

Ce qui différencie le plus les utilisateurs (premier axe factoriel de l'ACM) est leur inscription sur LinkedIn/Viadeo: entre ceux qui y sont inscrits et ceux qui ne les connaissent pas. Les premiers les utilisent professionnellement pour échanger avec des collègues, regarder ce que font les autres, mais aussi pour échanger avec des amis. Dans une moindre mesure, ils utilisent l'outil de réseau social interne à l'entreprise. Le second axe factoriel oppose les répondants sur l'emploi de Facebook. D'un côté, ceux qui sont inscrits sur ce réseau s'en servent pour des activités privées diverses (ex.: partager des photos, échanger des messages avec des amis hors du travail). Ils peuvent être présents sur Twitter, mais sont absents du réseau social interne à l'entreprise. D'un autre côté, ceux qui n'ont pas l'usage de Facebook ou n'y sont pas inscrits. Enfin, le troisième axe factoriel oppose ceux qui sont inscrits sur le réseau social interne de l'entreprise pour des usages professionnels et, dans une moindre mesure, pour des échanges personnels avec les collègues de travail, ainsi que pour regarder ce que font les autres, à ceux qui ne sont pas sur le réseau social interne. Chaque outil de réseau est ainsi lié à une pratique, professionnelle ou personnelle.

La Classification Ascendante Hiérarchique (mêmes variables actives) affine ce constat. Elle fait ressortir sept classes, allant des faibles utilisateurs, inscrits uniquement sur le réseau interne, aux forts utilisateurs, multiréseaux. Nous les avons regroupées en trois méta-classes, représentant à trois grands types d'utilisateur :

1. les multiconnectés (classes 1 et 2),

2. les usagers à titre personnel (classes 4 et 5 ),

3. les usagers à titre professionnel (classes 6 et 7 ).

La classe 3, hybride, est présentée à la fin : des individus ayant un usage avant tout personnel des outils de réseau social, mais qui estiment difficile de ne pas s'afficher professionnellement sur le réseau interne. Par leurs caractéristiques socioprofessionnelles, ils appartiennent aux multiconnectés, mais avec des comportements proches, respectivement des usagers à titre personnel (méta-classe 2) et des usagers à titre professionnel (classe 7).

\section{Les classes}

Les variables «illustratives" en italique caractérisent les répondants (statut professionnel, âge, représentations des réseaux sociaux, activité sur Internet), mais n'interviennent pas dans la construction des classes. Ce sont les usages (variables actives) qui définissent les classes. 


\section{Les multiconnectés (classes 1 et 2, 19,7 \% de l'échantillon).}

La première méta-classe regroupe de forts utilisateurs d'outils multiples, pour des raisons professionnelles mais aussi, de façon moins prédominante, personnelles. Elle est la plus proche des discours véhiculés sur les usages de ces outils, mais représente une minorité de notre échantillon (moins de $10 \%$ de l'ensemble des répondants).

L'utilisation des réseaux sociaux numériques permet d'améliorer leur carnet d'adresses professionnel, d'accéder au profil d'une personne sur les réseaux facilitant le contact. Les personnes des domaines d'activité communication, marketing, innovation y sont surreprésentées, sont des cadres à plus de $80 \%$, âgés de plutôt moins de 40 ans $(60 \%$ répartis entre les 20-30 et les 30-40).

On distingue deux sous-catégories : les multiconnectés et les connectés.

- Les multiconnectés permanents (5,7\% de l'échantillon) :

Utilisateurs de tous les outils de réseau, dont Facebook, surtout pour des activités professionnelles, ils se distinguent principalement par leur usage de Twitter, pour des raisons professionnelles et personnelles.

Des connectés en permanence sont surreprésentés ici, notamment via le téléphone mobile, dès qu'ils lancent Internet ; ils consultent (et interviennent) sur des forums, blogs, wikis externes au groupe de l'entreprise et se déclarent très à l'aise sur leurs compétences en informatique. Ils sont diplômés bac +5 et plus à plus de $60 \%$.

- Les connectés (14\% de l'échantillon) :

Utilisateurs de LinkedIn/Viadeo, pour des raisons professionnelles (et dans une moindre mesure, personnelles), ils sont présents sur Facebook pour des activités personnelles. S’ils connaissent tous l'outil Réseau interne, une petite majorité y est inscrite et seuls $30 \%$ l'utilisent.

Ils se connectent surtout de chez eux et pour un tiers d'entre eux (significativement plus que la moyenne) lors des pauses au travail. Ils consultent (et interviennent) aussi sur des forums, blogs, wikis externes au groupe et sont très à l'aise sur leurs compétences en informatique.

- Les usagers personnels "uniquement«(classes 4 et 5, 44,7 \% de l'échantillon).

À l'opposé, ces individus ont des usages privés restreints à Facebook et qu'ils protègent en ignorant les sollicitations professionnelles. Cela représente une grande partie des utilisateurs des réseaux sociaux, renforçant l'idée que ces outils sont perçus avant tout comme non professionnels.

$40 \quad$ Les réseaux n'aident pas pour les compétences professionnelles. Ils ne sont pas cadres (pour $55 \%$ ), peu diplômés ( $1 / 3$ au niveau bac +1 ou $+2,1 / 3$ bac ou inférieur). Ils sont plutôt affiliés au domaine «client» (30\%) de l'entreprise (et au domaine maintenance et déploiement de l’infrastructure réseau pour la première sous-classe).

$41 \quad$ On distingue deux sous-classes :

- Les usagers personnels (30,0 \% de l'échantillon). ont moins de 30 ans. 
- Les usagers personnels exclusifs (14,7\% de l'échantillon). 
Illustration 4 : Les répondants, selon le nombre de réseaux auxquels ils sont inscrits, sur l'utilité perçue des réseaux sociaux en ligne pour gérer des contacts

Ces outils autorisent à suivre des gens :

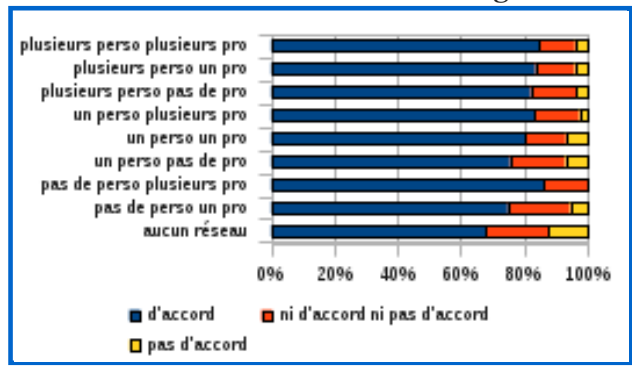

Illustration 5 : Les répondants, selon le nombre de réseaux auxquels ils sont inscrits, sur l'impact perçu des outils de réseau social sur l'organisation du travail

Ces outils permettent de rencontrer de nouvelles personnes :

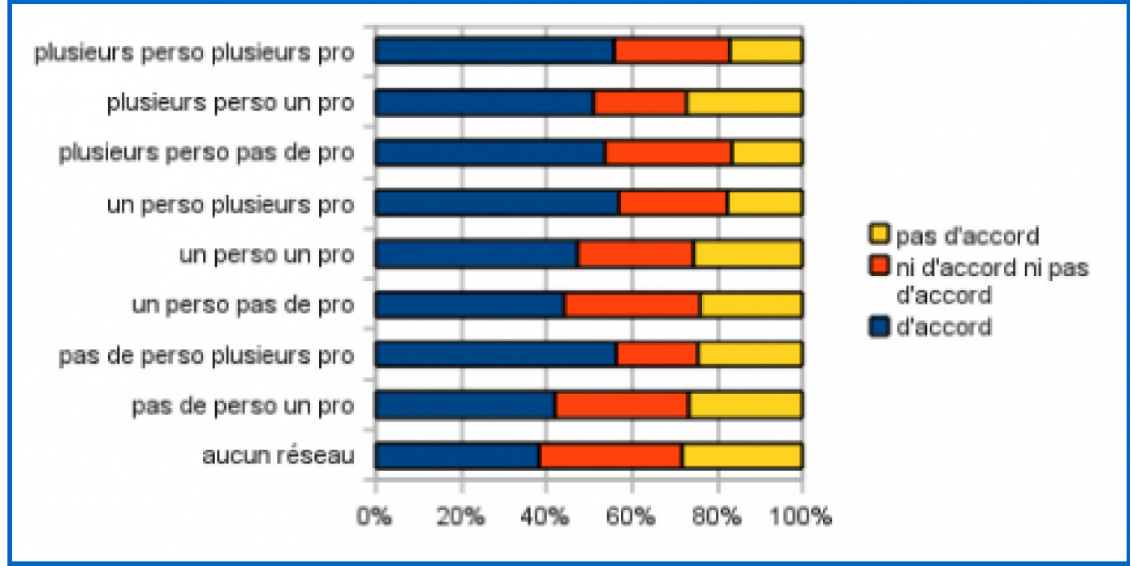

Ces outils sont un moyen de rester en contact avec vos connaissances « réelles »

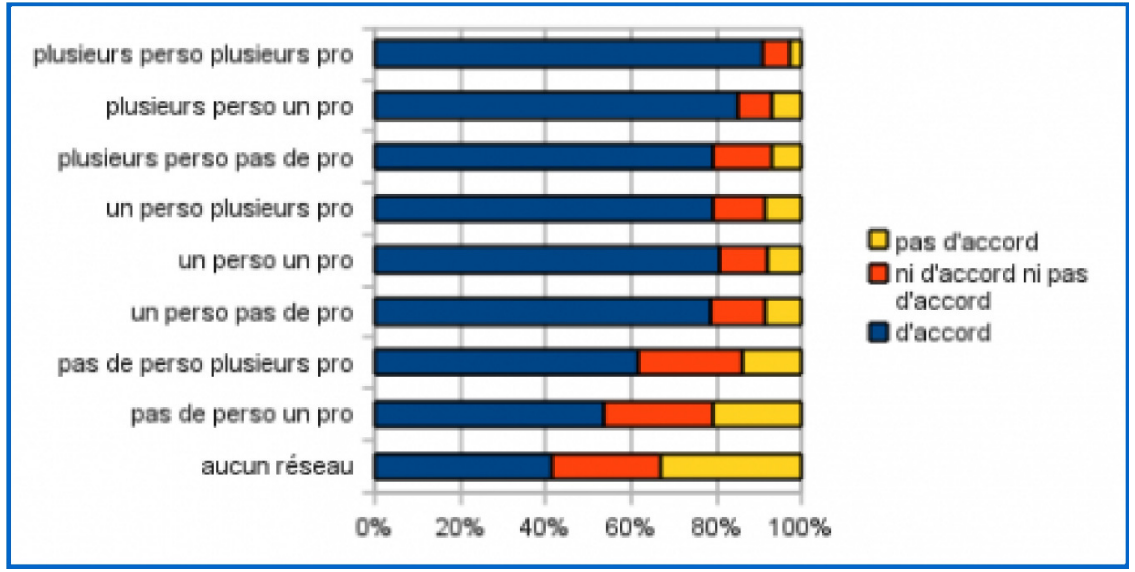

Ces outils vous aident à entretenir votre carnet d'adresses professionnel : 


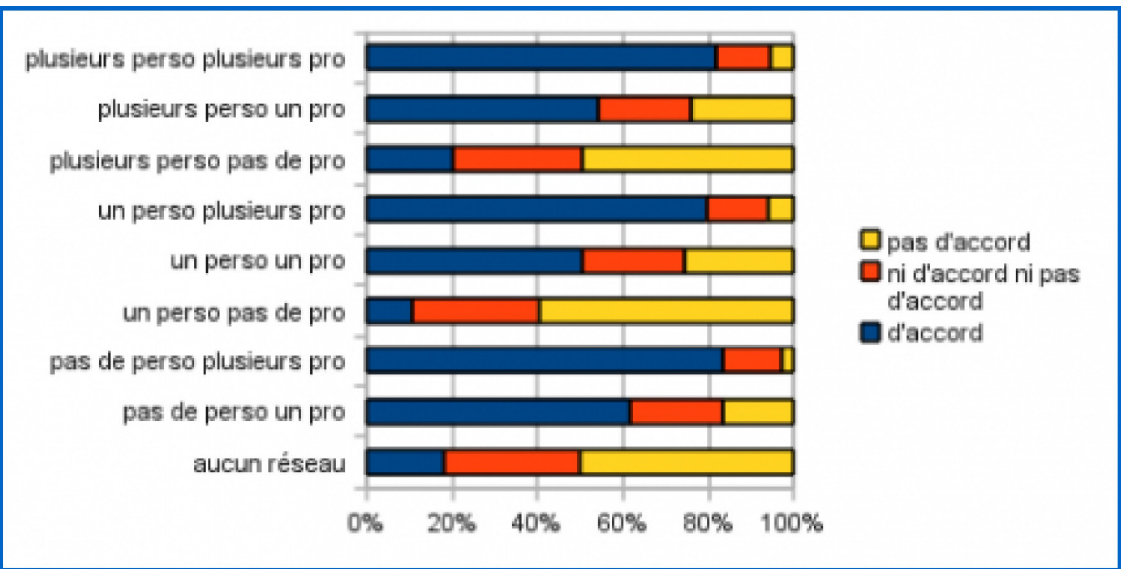

57

Le fait d'être "ami » avec des supérieurs sur les réseaux diminue la barrière hiérarchique :

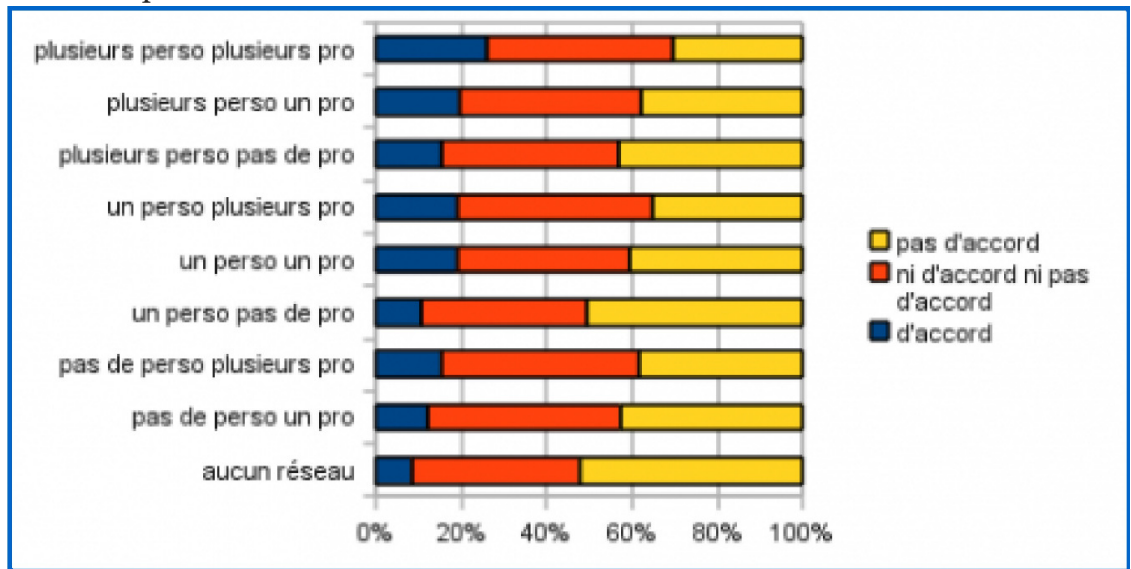

Accéder au profil d'une connaissance professionnelle facilite le contact :

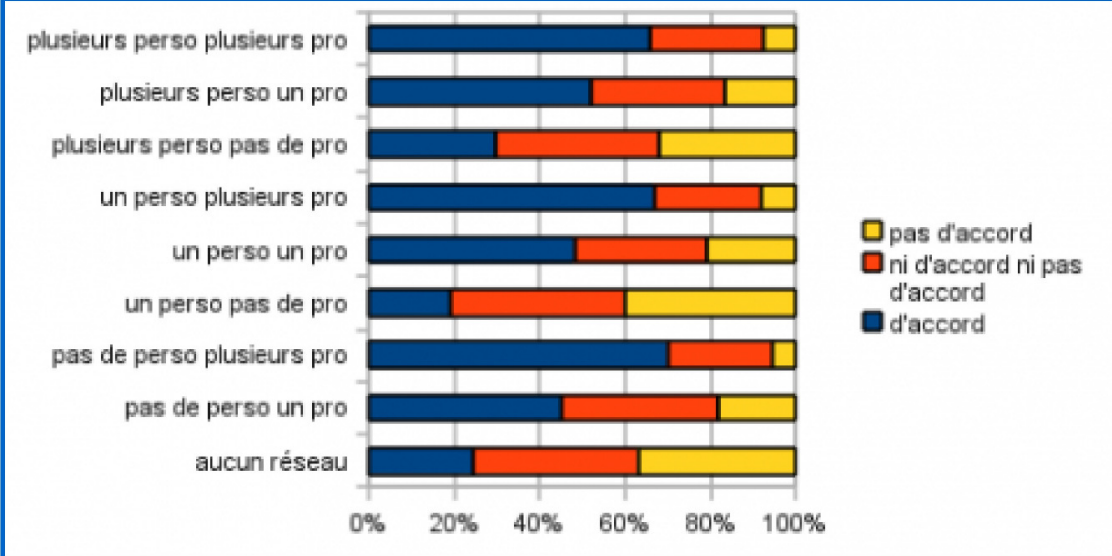

Ces outils augmentent votre efficacité professionnelle : 


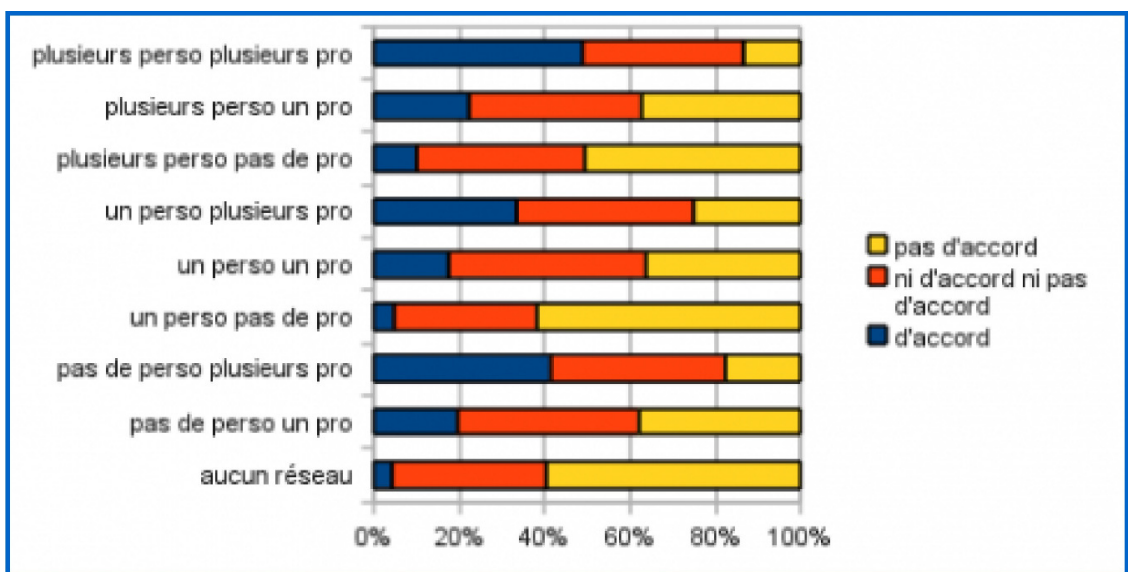

Cependant, cela n'empêche pas l'usage de l'outil, justifié par la position professionnelle, à des fins de veille ou de contrôle. Le statut des données produites sur ces sites est celui d'informations visibles, ce qui revient à ne publier «que ce que l'on veut bien rendre visible ». Deux grands principes ressortent de l'analyse des entretiens : un principe de nonusage et une gestion active des contacts.

\section{Des règles d'usage}

\section{Du non-usage des fonctionnalités du Web 2.0}

En plus de restreindre l'utilisation à certains outils, les enquêtés choisissent de restreindre les potentialités de l'outil, en refusant, par exemple, de l'utiliser dans sa dimension conversationnelle, dialogique. L'usager lit, consulte, suit, mais il n'interagit pas. Il a recours au courriel, mais pas à la messagerie du réseau, il ne partage ni ne poste d’informations ou très peu, hormis la mise à jour de son profil. Il ne publie pas sur les groupes auxquels il est inscrit, ses activités sont celles des « veilleurs ". C'est dans ce contexte qu'un outil, comme Facebook, peut être complètement écarté. La méfiance génère des pratiques en négatif, des choses à «ne surtout pas faire » : partager des photos, des informations de la vie privée, publier ses coordonnées. Le choix d'outils est pesé. Parmi les enquêtés, nombreux sont ceux qui déclarent déposer des photos qu'ils ont faites, les partager avec quelques-uns via un accès privé sur un site spécifique (Picasa, Flick’r) ou utiliser des sites « maison », également avec un accès réservé :

«C'est moi qui vais partager les photos que j'ai prises à un certain nombre de contacts»

« C'est moi qui donnerais l'accès aux gens que je connais » (RH, + 40 ans).

L'action de ceux qui, en revanche, publient, postent des commentaires ou interagissent via ces réseaux, est loin d'être libre: la parole est cadrée, contextualisée, filtrée par une évaluation systématique de toute publicisation, afin "de ne pas prendre de risques inconsidérés » (Ingénieur, + 50 ans) . Les principaux critères d'évaluation du statut de l'écrit sont relatifs au contenu, à la forme ou à la destination. Le principe de la visibilité induit une séparation du professionnel et du privé : ne pas inscrire des données d'état civil ou familiales, son affiliation politique, religieuse, sexuelle, etc.

De la même manière que « le privé », le travail peut faire partie des thèmes rejetés, pour ne pas risquer une mésinterprétation ou déformation de ce qu’on a « dit ».

« Et les questions qu'on peut me poser, c'est pas des questions où je veux répondre par écrit. Je suis très vigilant. (...) je ne laisserai pas interpréter, déformer des propos que j'ai pu écrire sur l'entreprise. (...) Donc je discute plus sur des forums de discussion sur (l'entreprise), le côté professionnel, côté technique, de soutenir des produits, de 
renseigner sur des informations ou des trucs comme ça. (...) Parce que je suis aussi un utilisateur comme les autres (...) Mais je ne parlerai pas de (l'entreprise) au sens

entreprise générale. » (RH et SI, + 40 ans).

Lorsque ce thème est abordé, il s'agit de la production du travail : des nouveaux produits, des innovations. Il existe des exceptions, comme le souligne un manager renvoyant à une situation précise de régulation des avis d'internautes sur leurs expériences avec des services d'entreprise:

«pour qu'ils mesurent leurs propos et qu'ils prennent conscience de l'impact que cela peut avoir pour le pauvre gars dans son centre d'appels. » (Manager, F, + 40 ans) .

\section{Un tri des contacts selon les usages et réseaux}

D'un point de vue professionnel, nous avons vu avec l'enquête statistique que ces outils constituent d'abord des supports pour entretenir un réseau de connaissance existant, plutôt que pour rencontrer de nouvelles personnes (voir les deux premiers graphiques de l'illustration 4). Pour ce faire, les interviewés créent des normes de gestion des contacts : ils peuvent être sollicités, acceptés, refusés ou supprimés ; ils sont organisés et assemblés dans des listes différentes. Les critères appliqués soulignent la variabilité des normes, selon les usages et les contenus publiés, selon les destinataires (connus ou pas), en fonction de la nature des liens (forts et proches ou faibles) et des outils utilisés. Trois procédures sont identifiées :

- la vérification,

- le fait de connaître ou pas ses contacts,

- un accord tacite sur les modes d'usage de l'outil ou normes de «bon comportement » en ligne.

Il n'est pas rare, lors d'une sollicitation de "regarder de qui il s'agit et quel intérêt (on) peu (t) y trouver » (Manager, $\mathrm{H},+40$ ans). La recherche via des moteurs de recherche est fréquente : regarder s'il y a suffisamment d'informations sur une personne ou pour préparer un échange, cela "permet aussi d'optimiser le temps de la rencontre » (Ingénieur, + 50 ans). Si la personne n'a aucun rapport avec soi, aucun lien même grossier, elle sera refusée. Si elle est connue par le biais de quelqu'un, elle peut être acceptée. Si la chance d'échanger semble trop ténue, le contact ne se fera pas, dès lors qu'on "ne le fait pas pour avoir une carte de visite » ou qu'on déclare " ne pas avoir besoin de cet affichage-là » (RH, + 40 ans), il n’y a pas d'intérêt à cumuler des contacts «virtuels ». Ceux-ci sont conçus comme des individus avec lesquels on a des liens, des échanges directs. Les connaître est bien sûr le critère par excellence d'entrée dans le réseau numérique.

Mais il se décline en de multiples combinaisons. Interviennent conjointement des indications d'orientation de l'usage selon l'outil. L'usage exclusivement personnel et privé d'un réseau social comme Facebook favorise, pour les interviewés, le lien avec des personnes connues dans la vie réelle (famille et amis, mais pas les amis des amis) et le refus systématique des sollicitations d'étrangers à cette sphère. Cette règle énoncée comporte quasiment toujours une ou quelques exceptions, encore que ce dérobement à la règle ne soit pas relatif à l'usage personnel, de loisir ou citoyen, mais au statut du contact. On peut donc avoir parmi ses « amis » un ou quelques collègues, mais « uniquement s'il y a un autre lien qu'une relation strictement professionnelle », le partage de goûts musicaux ou culturels, des centres d'intérêts communs "plus construits qu'une simple relation professionnelle » $(\mathrm{RH},+40$ ans). C'est donc le lien qui crée les échanges et non pas l'outil ; des échanges commencés en dehors du réseau numérique. Des collègues peuvent donc avoir «leur place là-dedans ». Sur un outil tel Facebook, les interviewés soulignent que leurs contacts sont connus dans la vie réelle, ou «les avoir rencontrés au moins une fois » (Doctorant, - 30 ans ). 
Quand l'usage est professionnel, sur des réseaux dédiés, le choix des contacts n'est pas moins pesé, mais « la porte est plus grande ouverte ». Un manager (+ $40 \mathrm{~ns})$ relève que sur LinkedIn, la distribution des contacts, connus environ à $80 \%$, forme des cercles relationnels : le premier est celui des anciens élèves, dont on accepte le contact au titre d'un corporatisme, particulièrement celui de grandes écoles d’ingénieurs.

« On est une communauté qui peut être amenée à s'aider ». "Donc, ceux-là, je les accepte, pas forcément en connaissant les gens, mais au titre du fait qu'on était sur les mêmes bancs. » (Manager,+ 40 ans).

Le deuxième maintient des liens avec des anciens collègues avec lesquels une relation en dehors du professionnel était établie et sans échange sur des projets actuels. Concernant les gens dans l'entreprise (le troisième cercle relationnel), un tri est effectué, mais il peut être léger. Sans aller à la "pêche aux contacts », des gens non connus peuvent venir à soi : " Si c'est quelqu'un qui vient chercher une expérience, on peut avoir de quoi échanger »(Manager, H,+4Oans) . La métaphore du bureau sur LinkedIn est la prolongation de pratiques effectives hors des outils numériques.

« Pour moi, dans LinkedIn, je suis au bureau si vous voulez. Au bureau, parfois j'ai la porte ouverte, parfois j'ai la porte fermée. Avec la porte ouverte, s'il y a un jeune ou un moins jeune qui passe et frappe et qui dit 'Bonjour (...), est-ce qu'on pourrait discuter cinq minutes ?' Là je dis 'Mais bien sûr, tu rentres', on va boire un café dans le bureau et s'il a des questions à poser, il pose des questions » (Manager, +40 ans).

La sélection des contacts est donc fonction à la fois des outils de réseau numérique et de l'orientation choisie de l'usage à caractère professionnel ou personnel et privé, du type de liens et des informations partagées. Parmi les interviewés, il semble que le choix des outils et de plusieurs comptes séparés (avec une identité spécifique), soit une technique de protection contre les mélanges des différents mondes auxquels ils appartiennent.

« Pour moi, Twitter c'est du réseau faible. (...) Facebook c'est plutôt du réseau fort. Viadeo c'est... je ne sais pas. Je ne sais pas, c'est un mélange. Et LinkedIn c'est un mélange. Sachant que ce sont plutôt des gens que vous avez rencontrés en fait, parce que si j'analyse un petit peu... c'est rarement des inconnus sur ces réseaux-là, quand même, LinkedIn, Viadeo, c'est des gens que j'ai rencontrés professionnellement ». (...) Il n'y a que de temps en temps que j'accepte un inconnu, mais c'est rare. J'aime pas trop en fait. Tandis que sur Twitter, j'accepte des inconnus. Sur Facebook, jamais !» « Sur Twitter, ça n’a aucune importance, au contraire » (Manager, + 50 ans).

« Twitter, typiquement, c'est un abonnement plutôt qu'une relation d'ami à ami. Enfin, l'inscription à un contact n'est souvent pas bidimensionnelle. Ça ne va pas forcément dans les deux sens, on peut s'inscrire au flux de quelqu'un sans que celui-ci souscrive au nôtre. (...) C'est plutôt l'identification de quelqu'un qui a les mêmes centres d'intérêt et qui poste des choses, qui, a priori, seraient intéressantes. C'est plutôt comme une souscription à un fil RSS, plutôt qu'à une personne en tant que telle » (Doctorant, 30 ans).

La sélection des contacts n'est pas définitive. Elle met en jeu un accord tacite, de confiance entre les personnes reliées, sur les modalités d'usage des outils. Des contacts peuvent être supprimés si aucune interaction n'a eu lieu ou si la confiance est mise à l'épreuve par un comportement virtuel non apprécié : comme le 'repost' de photographies personnelles et privées par des personnes connues. La figure du passager clandestin est aussi critiquée ou des contacts dont on "n'aime pas la vie virtuelle qu'ils mènent », parce qu'ils publient des contenus partiaux à caractère religieux, politiques ou personnels, et auxquels on n'a "pas envie forcément d'être identifié (...) » (Doctorant, - 30 ans).

Tous ces (non-)usages et ces règles soulignent en même temps l'intérêt et la difficulté d'usage des outils de réseau social pour ces salariés, qui sont bien conscients qu'il est nécessaire d'être présent sur ces réseaux, et qu'il est difficile de maitriser complètement la 
segmentation des usages et des outils et la présentation numérique de soi qu’ils réalisent.

\section{Les frontières floues de l'identité}

Les plus nombreux, notamment parmi les cadres, soulignent l'importance d'être présents sur le Web et justifient ainsi la publicisation d'informations sur soi : "De mettre des choses qui ont du sens », "des informations maîtrisées » (Manager, + 40). Nous l'avons souligné, ce ne sont pas les novices, mais les plus aguerris à ces usages qui disent la difficulté à gérer l'identité numérique. Cette gestion prend la forme d'un travail de contrôle de l'information sur soi : la capacité à s'exposer tout en contrôlant son exposition, ce qui renvoie aux stratégies évoquées. Une capacité qui, comme l'a mis en avant D. Cardon (2008), « réclame des compétences sociales et relationnelles spécifiques" qui sont "très inégalement distribuées ".

Dans la même perspective, le travail de présentation de soi, professionnel et visible sur les sites de réseau social, constitue une autre stratégie pour présenter un portrait de soi. Dans ce cas, il s'agit beaucoup plus de "montrer un peu plus ce qu' (on) est en tant qu'homme professionnel » (Manager, + 40 ans), enrichir les traces de son identité en ligne. Les profils habituels sont considérés comme froids et l'image de soi doit être cohérente entre les différentes sources d'information. Un interlocuteur témoigne avoir enquêté sur son identité numérique auprès de ses contacts sur ces réseaux. Cette démarche, soutenue par un coach, a permis de questionner ce qu'il veut montrer, qui il veut être, "qui il est » et dans quelle direction il veut aller: "C'est une démarche qui permet de construire », conclut-il (Manager, + 40 ans). D'autres actions de régulation de son image numérique peuvent consister à réduire son empreinte sur le Web ou à la cacher (recourt à l'anonymat par l'utilisation de pseudonymes sur certains sites et forums auxquels on participe et où les informations sont partagées avec tous, brider un profil ou effacer des traces, et pour les aguerris, surveiller les plateformes via des agrégateurs comme 'Flavors me').

Finalement les divers usages sur les nombreux outils de réseau social participent également de la fabrication d'une image, qui peut être chaque fois différente. La démultiplication de ces images numériques de soi peut alors, par voie de conséquence, alimenter la description d'un phénomène de « dissolution de l'identité » (Cardon, 2008).

« C'est pour ça que j'emploie le terme d'ombre numérique, en fait. C'est un jeu

d'ombres, un jeu d'acteur un peu. (...). Je ne peux pas avoir la même image » (Manager,

+50 ans).

En avoir conscience ne signifie pas, malgré toutes les précautions prises, que l'on soit à l'abri de toute erreur de jugement ou d'appréciation. Tout questionnement relatif aux outils ne peut pas se réduire à sa seule dimension technique. Le paramétrage et la maîtrise de la technicité ne sont qu'une des dimensions de la maîtrise de l'usage. Cette dernière réclame d'autres compétences qui, elles, sont moins faciles à acquérir. Elles sont moins évidentes, plus floues ou imperceptibles. Elles font appel premièrement, aux représentations relatives à la séparation des mondes sociaux, dont les frontières sont rarement aussi nettes dans la vie réelle (et/ou virtuelle) qu'elles ne le sont en théorie, et deuxièmement, à l'évaluation du statut des informations publiées, enfin troisièmement, à la définition et donc structuration des types de relation avec les contacts.

Les usagers font preuve d'une réflexivité de leur action et d'un autocontrôle, valorisant plutôt des actions réfléchies de partage, d'échange d'avis posés et pesés.

« Je ne veux pas de problème, je ne veux pas de polémique, je ne veux pas avoir des avis divergents des autres, où on se crêpe le chignon parce que dans la vraie vie, on se crêpe le chignon et du coup, j'ai pas besoin de ça sur le Net. Les ennuis, on en a assez dans la vraie vie pour ne pas, en plus, en créer... Oui, qu'on aille les alimenter sur le Web » (Manager, +40 ans).

« Je fais attention à tout ce que je dis »; « J'ai fait un blog pour des raisons très 
précises et je fais attention à ce que j'y publie. Je ne fais pas partie des fous qui écrivent tout et n'importe quoi, n'importe comment. C'est un outil » (Manager, + 40 ans).

Le problème de la frontière privée/professionnelle est un des sujets récurrents des discours recueillis. Si les outils n'ont pas créé ce brouillage des sphères, ils y contribuent ; leur mobilité et leur portabilité introduisent notamment de nouvelles temporalités et de nouveaux espaces dans la production du travail.

« Le fait qu'aujourd'hui, on a des laptops qu'on peut emmener n'importe où, le fait que même, quand il y a une réunion importante, on peut quand même prendre une semaine de vacances parce qu'on peut se connecter par téléphone et avoir accès à son mail, etc. » (Manager, +40 ans).

« J'ai lu un article récemment, (...) le temps de présence au bureau était un temps de détente et que je travaillais plus à domicile, chez moi (...). Alors, dans ce cas-là, il y avait plus de deux tiers des cas pour lesquels c'est comme ça. Alors, effectivement, lorsque vous travaillez sur un dossier etc., ce n'est pas du boulot ! C'est en open space, c'est extrêmement difficile de se concentrer en permanence. Vous demandez à ne pas être dérangé, vous êtes dérangé. (...) et j'ai remarqué qu'il y avait vraiment des sujets qui me tenaient à cœur et qu'il fallait que j'avance, je les travaille chez moi. Je pense que c'est une majorité des cas qui est pareille. Par contre, pour interagir, pour (...) c'est au travail. Sur des sujets où il faut approfondir, c'est rarement au boulot (...). Au boulot, je vais peut-être digérer ce que j'ai comme informations » (Manager, + 50 ans).

La place que ces technologies occupent dans la recomposition des temps sociaux et des liens de sociabilité, ainsi que la question de l'évolution des usages par un même acteur, sont à considérer. Ainsi lorsque des contacts professionnels deviennent des amis sur Facebook, la frontière des sphères privée et professionnelle n'est plus aussi tangible.

«Et ça, j'ai un peu de mal à le gérer parce que je vous le dis, entre les photos de vacances et puis, l'échange sur les sujets purement business, c'est un peu dur de gérer le truc. Je pense que ça veut dire qu'on dévoile à des contacts professionnels des choses, pas qu'on n'a pas envie de dévoiler, mais des choses qu'on ne s'attendait pas à dévoiler » (Manager, +40 ans) .

L’injonction plus ou moins forte sur le marché de l'emploi à être présent sur ces outils de réseau social participe de la réorientation des usages à des fins professionnelles et de réseautage.

« Enfin à l'origine, c'était uniquement professionnel, aujourd'hui, ça devient aussi un peu personnel. J'ai encore un petit problème à faire la frontière entre le professionnel et le personnel parce que ces outils vont permettre à la fois de retrouver vos copines de classe que vous avez perdues de vue et aussi d'avoir des informations à usage professionnel, de faire votre propre empreinte digitale, vos empreintes numériques pour votre propre compte, en particulier sur LinkedIn ou Viadeo. Je sais que, par exemple, pour un certain nombre d'entretiens de recrutement, par exemple, le recruteur vous demande quel est votre réseau et dans quel secteur. Alors, il faut l'avoir constitué avant son réseau. » (Manager, +40 ans).

Ce qui peut aboutir à un déplacement des lignes, voire à leur suppression. Le temps de gestion peut être coûteux et dépasse largement celui de l'apprentissage des paramétrages techniques indispensables à la relative protection des données personnelles ou privacy.

« Non, je pense que je n’y arrive pas très bien. Je ne suis pas très fort. Je pense que je pourrais mieux faire et en passant un peu plus de temps, en réglant... C'est même pas une question de réglage de mes paramètres de sécurité, c'est pas ça, c'est plus que... de temps en temps sur le Web, quand on est caché par un pseudo, on peut dire des choses qu'on ne dirait pas si on était en face-à-face avec une personne, qui est notre collègue. » « Et c'est un collègue qui est connecté à vous, au moment où vous le publiez, vous agissez de façon spontanée comme ça, dans l'instant, sans vous souvenir que c'est votre voisin de bureau, il va savoir que, je ne sais pas quoi, le fait que vous avec votre cousin Tartempion... Enfin, sur un sujet tout à fait personnel, qui ne regarde que 
vous. » (Manager, + 40 ans). 
maintenir une distinction entre la vie privée et l'activité professionnelle, forcément plus exposée, plus publique. Cela passe essentiellement par une segmentation des usages et des contacts par les outils, qui sont identifiés comme servant des mondes différents (Facebook pour les amis, LinkedIn ou Viadeo pour le travail). Les sites de réseaux sociaux apparaissent " comme un moyen additionnel de rencontrer de nouvelles personnes et donc de renforcer une vie sociale le plus souvent déjà riche dans le monde réel » (Lethiais, Roudaut, 2010). Réaffirmer une identité professionnelle, dans le monde du travail, en retrouvant un rôle d'expert, est un fait observé, mais ce type de reconquête rendu possible par l'utilisation des outils de réseau social semble moins fréquent. Si l'entreprise est toujours présente, en revanche, elle ne constitue plus vraiment une frontière organisationnelle et professionnelle, qui s'impose aux pratiques de ses salariés. Les réseaux et les frontières, notamment professionnels, sont gérés à un niveau individuel.

La porosité de ces réseaux, des sphères (privée, amicale, professionnelle) dans la vie réelle, qui entraîne une difficulté à cloisonner les espaces de socialité, se retrouve sur ces outils. Actifs dans leur relation à la technique, les individus construisent leur usage en développant des "comportements destinés à se protéger », ce qu'avaient déjà relevé $\mathrm{E}$. Kessous et B. Rey (2009: 53), mais ils se laissent aussi guider par le contexte, des envies, des dynamiques d'interaction. Émergent alors des exceptions aux règles qu'ils ont construites, des failles dans le cadre a priori bien structuré de leur pratique, qui sont catalysées par le caractère public des outils : manquements à l'évaluation du statut de l'information publiée ou de son interprétation, étourderies ou gaffes dans la diffusion d'une information à des destinataires inopportuns. Tous ces menus faits, issus de l'actioninteraction, rendent la structuration de l'usage vulnérable, conséquence d'un " entrelacement des usages », mais aussi «des médias », tel que l'avaient décrit Cardon et al. (2005).

S'ils ont conscience de ce risque, les individus qui se connectent, affichent leurs activités, voire s'exposent, se disent "prêts » à le prendre, car la valeur estimée de cette forme de visibilité dépasse a priori le coût du risque7. Injonctions de l'entreprise, évolution du travail, de ses formes et modes d'organisation, somment les acteurs sociaux à se montrer " soi en tant que professionnel », dans un monde professionnel lui-même jugé incertain, où la mobilité et la remise en cause permanente sont une norme, " où les valeurs importantes sont l'engagement personnel et l'entretien permanent des relations ${ }^{8}$, non loin du «travail créateur » analysé par P.-M. Menger (2009).

\section{Bibliographie}

Bateman, P.J., Pike, J.C. \& Butler, B. S. (2011). To disclose or not: Publicness in social networking sites. Information Technology \& People, 24 (1) : 78-100.

Beaudouin, V. \& Velkovska, J. (1999). Constitution d'un espace de communication sur Internet (forums, pages personnelles, courrier électronique...). Réseaux, 17(97) : 121-177.

Beaudouin, V., Cardon, D. \& Mallard, A. (2001). De clic en clic : Créativité et rationalisation dans les usages des intranets d'entreprise. Sociologie du Travail, 43 (3) : 309-326.

Benghozi, P.-J. (2000). Le développement des NTIC dans les entreprises françaises: Premiers constats. Réseaux 104(2000/6) : 31-57.

Boltanski, L. \& Chiapello, E. (1999). Le nouvel esprit du capitalisme. Vol. 2, Gallimard, Paris.

Butler, B., Sproull, L., Kiesler, S. \& Kraut, R. (2007). Community effort in online groups: Who does the work and why?. In Weisband, S. (Ed.), Leadership at a distance: Research in Technologically Supported Work. Lawrence Erlbaum, Mahwah, NJ.

Brzozowski, Michael J. (2009). WaterCooler: exploring an organization through enterprise social media. In Proceedings of the ACM 2009 International Conference on Supporting Group Work. New York, NY, USA: ACM, pp. 219-228.

Cardon, D. \& Smoreda, Z. (2014). Réseaux et les mutations de la sociabilité. Réseaux 32(184-185) : 161-185.

Cardon, D. (2008). Le design de la visibilité : Un essai de typologie du web 2.o. InternetActu.net. Consulté le 04/05/2017. 
Cardon, D., Smoreda, Z. \& Beaudouin, V. (2005). Sociabilités et entrelacement des médias. In Moati, $\mathrm{Ph}$. (Ed.), Nouvelles technologies et modes de vie : Aliénation ou hypermodernité ?, pp. 99-123.

Cardon, D. (2000). La production coopérative des factures. Cas de mise en place d'un outil de groupware. Réseaux 104(2000/6): 95-118.

Datchary, C. (2008). Gérer la dispersion : un travail collectif. Sociologie du Travail, 50(3): 396-416.

Demazière, D., Horn, F. \& Zune, M. (2009). Sociabilité et contrôle social dans les collectifs de développement de logiciels libres. In Nicours, S. (Ed.), Le travail militant. Rennes, Presses Universitaires de Rennes, coll. « Respublica », pp. 117-126.

Denis, J. \& Assadi, H. (2005). Les usages de l'e-mail en entreprise. Efficacité dans le travail ou surcharge informationnelle? In Kessous, E. \& Metzger, J.-L. (Eds.), Le travail avec les technologies de l'information. Paris, Lavoisier, pp. 135-155.

Ellison, N.B., Steinfield, C. \& Lampe, C. (2007). The benefits of Facebook 'friends': Social capital and college students' use of online social network sites. Journal of Computer - Mediated Communication 12(4): $1143-1168$.

Georges, F. (2009). Représentation de soi et identité numérique. Réseaux 154(2009/2): 165-193.

Glynn, C.J., Huge, M.E. \& Hoffman, L.H. (2012). All the news that's fit to post : A profile of news use on social networking sites. Computers in Human Behavior 28(1): 113-119.

Granjon, F. \& Denouël, J. (2010). Exposition de soi et reconnaissance de singularités subjectives sur les sites de réseaux sociaux. Sociologie 1(1/20101): 25-43.

Granjon, F. (2011). De quelques pathologies sociales de l'individualité numérique. Réseaux 167 (2011/3): 75-103.

Gross, R. \& Acquisti, A. (2005). Information revelation and privacy in online social networks. In Proceedings of the 2005 ACM workshop on Privacy in the electronic society, pp. 71-80.

Guesmi, S. \& Rallet, A. (2012). Web 2.0 et outils de coordination décentralisée, Revue Française de Gestion 224(5/2012): 139-151.

Jauréguiberry, F. (2014). La déconnexion aux technologies de communication. Réseaux 186(2014/4): 17-49.

Joinson, A. (2008). Looking at, looking up or keeping up with people ? Motives and use of Facebook. In Proceedings of CHI 2008. ACM, New York, NY, USA, pp. 1027-1036.

Jullien, N., Roudaut, K. \& Le Squin, S. (2011). L'engagement dans des collectifs de production de connaissance en ligne. Le cas GeoRezo. Revue Française de Socio-Économie 8(2011/2): 59-83, dossier «Socio-économie du numérique ».

Karoui, M. \& Dudezert, A. (2012). Capital social et enjeux de pouvoir : une perspective socio-politique de l'appropriation d'une technologie de réseaux sociaux au sein d'une collectivité territoriale. Systèmes d'Information et Management 17 (1): 49-80.

Kaufmann, J.-C. (2004). L’invention de soi, Une théorie de l'identité. Paris, Armand Colin.

Kessous E. \& Rey B. (2009). Économie numérique et vie privée. Hermès-La Revue 1(53):49-54.

Kiesler, S. \& Kraut, R. (2007). Community effort in online groups: Who does the work and why? In Weisband, S. (Ed.), Leadership at a distance: Research in Technologically Supported Work. Lawrence Erlbaum, Mahwah, NJ.

Lancelot Miltgen, C. (2011). Vie privée et marketing : Étude de la décision de fournir des données personnelles dans un cadre commercial. Réseaux 167(2011/3): 131-166.

Le Douarin, L. (2007). C'est personnel! L'usage des TIC par les cadres dans l'articulation des temps sociaux : Vers une évolution de la rationalisation au travail ? L'Homme et la société 1-2(163-164): 7594 .

Lethiais, V. \& Roudaut, K. (2010). Les amitiés virtuelles dans la vie réelle. Réseaux 164(2010/6): 1349.

Mallard, A. (2014). Métamorphoses d'une question scientifique: Trente ans de recherches sur l’inscription des TIC dans les univers productifs. Réseaux 184-185(32): 35-69.

Menger P.-M. (2009). Le travail créateur : S'accomplir dans l’incertain. Paris, Gallimard-Seuil.

Metzger, J.-L. \& Cléach, O. (2004). Le télétravail des cadres : Entre suractivité et apprentissage de nouvelles temporalités. Sociologie du Travail 46(3): 433-450.

Moati, P. (2005). Nouvelles technologies et modes de vie : Aliénation ou hypermodernité ? Petite bibliothèque du Crédoc, Éditions de l'Aube.

Morris, M.R., Teevan, J. \& Panovich, K. (2010). What do people ask their social networks, and why ? A survey study of status message Q\&A behavior. In Proceedings of the 28th international conference on Human factors in computing systems CHI'10. New York, NY, USA. ACM, pp. 1739-1748.

Muhlmann, D., 2001. Des nouvelles technologies à l'image des vieilles organisations. Sociologie du 
Travail 43(3): 327-347.

Satchell, C. \& Dourish, P. (2009). Beyond the user: Use and non-use in HCI. In Proceedings of the 21st Annual Conference of the Australian Computer-Human Interaction Special Interest Group 'Design: Open 24/7'. OZCHI 'o9, New York, NY, USA. ACM, pp. 9-16.

Skeels, M.M. \& Grudin, J. (2009). When social networks cross boundaries: A case study of workplace use of Facebook and LinkedIn. In Proceedings of the ACM 2009 international conference on Supporting group work, GROUP ‘09, New York, NY, USA. ACM, pp. 95-104.

Ellison, N.B. \& Lampe, C. (2009). Bowling online: Social networking and social capital within the organization. In Proceedings of the fourth international conference on Communities and technologies, C\&T '09, New York, NY, USA. ACM, pp. 245-254.

Stenger, T. \& Coutant, A. (Eds.) (2011). Ces réseaux numériques dits sociaux. Hermès-La Revue 2011/1(59).

Thom-Santelli, J., Millen, D.R. \& Gergle, D. (2011). Organizational acculturation and social networking. In Proceedings of the ACM 2011 conference on Computer supported cooperative work, CSCW '11, New York, NY, USA. ACM, pp. 313-316.

Wenger, E. (1998). Communities of practice: Learning, Meaning, and Identity. Cambridge University Press, Cambridge, Mass.

Wenger, E., McDermott, R. \& Snyder, W. (2002). Cultivating communities of practice: A guide to managing knowledge. Harvard Business School Press, Cambridge, Mass.

Wu, A., DiMicco, J.M. \& Millen, D.R. (2010). Detecting professional versus personal closeness using an enterprise social network site. In Proceedings of the 28th international conference on Human factors in computing systems, CHI'1O. New York, NY, USA. ACM, pp. 1955-1964.

\section{Documents annexes}

- Annexe 1 : Présentation de l'enquête (application/msword - 39k)

$\bullet$

- Annexe 2 : L’inscription sur les outils de réseau social (application/msword - 132k)

$\bullet$

- Annexe 3. Analyse de données et classification sur les usages des réseaux sociaux numériques (application/msword - 137k)

\section{Notes}

1 Metzger et Cléach (2004) à propos du télétravail ; Le Douarin (2007) à propos du téléphone et du courriel.

2 Butler et al. (2007) pour les communautés de pratique en ligne ; Demazière et al. (2009) pour les communautés épistémiques en ligne.

3 Après élimination des réponses incomplètes, 8618 questionnaires ont été conservés, dont 4187 inscrits sur un réseau social. Ce questionnaire était à l'initiative du Comité d'Entreprise (CE) et accepté par la direction des ressources humaines. Sur demande du CE, la direction avait fourni aux chercheurs l'adresse électronique de l'ensemble des salariés (voir la présentation en annexe).

4 Ces outils sont d'abord adoptés par les jeunes (Glynn et al., 2012), et pour la France, enquête Ifop 2009.

5 Les métiers informatiques sont surreprésentés par rapport à la structure des emplois dans l'entreprise.

6 Les inscrits depuis plus de trois ans représentent 10,5\% des répondants ; le même pourcentage que ceux inscrits depuis moins d'un an.

7 Pour reprendre une des conclusions de Lancelot Miltgen (2011) sur le dévoilement des données personnelles dans un cadre commercial.

8 Pour citer Denis et Assadi (2005), en référence à un «nouvel esprit du capitalisme » (Boltanski, Chiapello, 1999).

Table des illustrations 


\begin{tabular}{|c|c|c|c|}
\hline & & Titre & Illustration 1 : Les usages suivant les outils \\
\hline & & URL & http://terminal.revues.org/docannexe/image/1610/img-1.png \\
\hline & & Fichier & image/png, 16k \\
\hline & & URL & http://terminal.revues.org/docannexe/image/1610/img-2.png \\
\hline & & Fichier & image/png, 10k \\
\hline & & URL & http://terminal.revues.org/docannexe/image/1610/img-3.png \\
\hline & & Fichier & image/png, 10k \\
\hline & & URL & http://terminal.revues.org/docannexe/image/1610/img-4.png \\
\hline & & Fichier & image/png, $11 \mathrm{k}$ \\
\hline & & URL & http://terminal.revues.org/docannexe/image/1610/img-5.png \\
\hline & & Fichier & image/png, $11 \mathrm{k}$ \\
\hline & & URL & http://terminal.revues.org/docannexe/image/1610/img-6.png \\
\hline & & Fichier & image/png, 9,1k \\
\hline & & URL & http://terminal.revues.org/docannexe/image/1610/img-7.png \\
\hline & & Fichier & image/png, 21k \\
\hline & & URL & http://terminal.revues.org/docannexe/image/1610/img-8.png \\
\hline & & Fichier & image/png, 21k \\
\hline & & URL & http://terminal.revues.org/docannexe/image/1610/img-9.png \\
\hline & & Fichier & image/png, 21k \\
\hline & & URL & http://terminal.revues.org/docannexe/image/1610/img-10.png \\
\hline & & Fichier & image/png, 22k \\
\hline 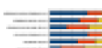 & & URL & http://terminal.revues.org/docannexe/image/1610/img-11.png \\
\hline & & Fichier & image/png, 22k \\
\hline$\overline{\overline{\underline{\underline{\underline{\underline{E}}}}}}$ & E... & URL & http://terminal.revues.org/docannexe/image/1610/img-12.png \\
\hline & & Fichier & image/png, 21k \\
\hline
\end{tabular}

\section{Pour citer cet article}

Référence électronique

Karine Roudaut et Nicolas Jullien, « Les usages des outils de réseau social par des salariés : Des registres privés et professionnels individualisés », Terminal [En ligne], 120 | 2017, mis en ligne le 03 mai 2017, consulté le 04 mai 2017. URL : http://terminal.revues.org/1610

\section{Auteurs}

Karine Roudaut

Chercheure associée au CRAPE-Arènes, M@rsouin, karine.roudaut@gmail.com

Articles du même auteur

Les enjeux de la numérisation et de l'ouverture d'archives : le point de vue des professionnels [Texte intégral]

L'exemple de la production et la diffusion d'un patrimoine local.

Paru dans Terminal, $110 \mid 2012$

« Je suis peut-être un pirate, mais je ne me sens pas du tout délinquant ! » [Texte intégral] Note de recherche

Paru dans Terminal, 115 | 2014

Nicolas Jullien

Maître de conférences HDR, IMT Atlantique, LEGO- M@rsouin, Nicolas.Jullien@telecom-bretagne.eu

Articles du même auteur

Regards sur le travail et les TIC [Texte intégral]

Entretien avec Jean-Pierre Durand

Paru dans Terminal, $110 \mid 2012$ 
Les usages des outils de réseau social par des salariés : Des registres privés et professi... Page 21 sur 21

Relations médiatisées par le numérique et outils d'apprentissages [Texte intégral]

Contributions du 12ème et 13ème séminaire M@rsouin

Paru dans Terminal, 117 | 2015

Pratiques des outils numériques et relations sociales [Texte intégral]

Contributions du 11e séminaire M@rsouin

Paru dans Terminal, 115 | 2014

L'industrie informatique dans la société de l'information [Texte intégral]

Paru dans Terminal, 113-114 | 2013

\section{Droits d'auteur}

tous droits réservés 